\title{
Aprendizagem de posturas em equoterapia por crianças com transtorno do espectro autista (TEA)
}

\author{
Learning of positions in hippotherapy by children with autistic spectrum \\ disorders (ASD)
}
Aprendizaje de posturas en equinoterapia por niños con transtorno del espectro autista (TEA)

\author{
* Gardenia de Oliveira Barbosa \\ Doutora na Universidade Federal de São Carlos, São Carlos, São Paulo, Brasil. \\ garativ@gmail.com

\section{** Mey de Abreu van Munster} \\ Professora doutora pela Universidade Federal de São Carlos, São Carlos, São Paulo, Brasil. \\ munster.mey@gmail.com
}

Recebido: 19 de junho de 2018

Aprovado: 17 de janeiro de 2019

\section{RESUMO}

O estudo analisou a efetividade dos níveis de auxílio físico-verbal, visual-verbal e verbal no processo de aprendizagem de posturas em equoterapia por crianças com Transtorno do Espectro Autista (TEA). O método foi baseado em delineamento de sujeito único, do tipo $A B$, envolvendo três crianças com TEA, com idades entre quatro e nove anos. Os participantes foram submetidos à intervenção equoterápica (31 sessões individuais de equoterapia) com duração de quatro meses, e frequência de duas vezes por semana. Como instrumentos de coleta de dados foram empregados: 1. anamnese preenchida junto aos pais ou responsáveis; 2. protocolo de Avaliação de Habilidades Básicas de Aprendizagem (Assessment of Basic Learning Abilities - ABLA); 3. diário de campo; 4. lista de checagem para registro diário de cada um dos auxílios utilizados em cada sessão de equoterapia. A análise dos dados referente à evolução de cada participante foi descritiva. Ao término da intervenção, todos os participantes foram capazes de realizar as posturas com auxílio exclusivamente verbal, sendo o processo de aprendizagem mediado pelos auxílios visualverbal e físico-verbal, sendo este último predominante. Assim, verificou-se que os níveis de auxílio necessários à aprendizagem de posturas em equoterapia devem variar conforme as necessidades individuais das crianças com TEA, não havendo um único método eficiente; todavia observa-se que a associação de estímulos de maneira direcionada pode levar ao sucesso na aprendizagem das crianças.

Palavras-chave: Educação Especial; Equoterapia; Autismo. 


\section{ABSTRACT}

The study analyzed the levels of physical-verbal assistance, visual-verbal assistance, and verbal communication in the process of learning positions in hippotherapy by children with Autistic Spectrum Disorder (ASD). The research method was based on a single subject design, type $A B$, involving three children with $A S D$ with ages between four and nine years. Participants were submitted to 31 individual sessions of hippotherapy, during four months, twice a week. Instruments used for data collection were: 1. Anamnesis filled in with parents or guardians; 2. Protocol for Assessment of Basic Learning Abilities (ABLA); 3. Field diary; 4. Daily recording checklist for each of the aids used during each session of hippotherapy. Descriptive analysis regarding the evolution of each participant was used. At the end of the intervention it was observed that all participants were able to perform all positions with exclusively verbal assistance. The learning process was mediated by visual-verbal and physical-verbal aids with the latter being more predominant. Thus, levels and types of aid needed to learn positioning will vary according to the individual needs of child with ASD, and there is no single efficient type of method; however, it is observed that the association of stimuli in a directed way can lead to success in the child's learning.

keywords: Special Education; Hippotherapy; Autism.

\section{RESUMEN}

El estudio objetivó analizar la efectividad de los niveles de auxilio físico-verbal, visual-verbal y verbal en el proceso de aprendizaje de posturas en equinoterapia por niños con Trastorno del Espectro Autista (TEA). En cuanto al método, el presente estudio se basó en delineamiento de sujeto único, del tipo $A B$; participaron del estudio tres niños con TEA con edades entre cuatro y nueve años. Los participantes fueron sometidos a la intervención equoterápica con una duración de cuatro meses, dos veces por semana, correspondiendo a 31 sesiones individuales de equinoterapia. Como instrumentos de recolección de datos fueron empleados: 1. anamnesis rellenada junto a los padres o responsables; 2. Protocolo de Evaluación de Habilidades Básicas de Aprendizaje (ABLA); 3. diario de campo; 4. lista de comprobación para el registro diario de cada una de las ayudas utilizadas en cada sesión de equinoterapia. El análisis de los datos referentes a la evolución de cada participante fue descriptivo. En cuanto a los resultados, se observó que, al término de la intervención, todos los participantes fueron capaces de realizar las posturas con ayuda exclusivamente verbal, siendo el proceso de aprendizaje mediado por las ayudas visual-verbal y físico-verbal, siendo este último predominante. Se concluyó que los niveles de ayuda necesarios para el aprendizaje de posturas en equinoterapia deben variar según las necesidades individuales de los niños con TEA, no habiendo un único método eficiente; sin embargo, se observa que la asociación de estímulos de manera direccionada puede llevar al éxito en el aprendizaje de los niños.

Palabras clave: Educación Especial; Hipoterapia; Autismo. 


\section{Introdução}

O transtorno do espectro autista (TEA) leva a inadequações na interação social, habilidade comunicativa, aprendizado e capacidade de adaptação. Manifesta-se antes dos três anos de idades, podendo a criança apresentar desenvolvimento típico até um ou dois anos (DSM 5, 2013).

As pessoas com TEA podem apresentar atraso ou ausência do desenvolvimento da linguagem falada; os que verbalizam podem apresentar dificuldades para iniciar e manter uma conversação, sendo possível que apresentem também ecolalia ou linguagem idiossincrática (DSM 5, 2013).

Os distúrbios do comportamento manifestados por pessoas com TEA frequentemente estão associados à ausência ou dificuldades graves na comunicação e interação com o outro, levando a condutas agressivas e inadequadas. Segundo Walter (2006) inabilidades na comunicação verbal podem levar a atitudes comportamentais agressivas ou inadequadas, devido à dificuldade de se estabelecer um canal de comunicação comum, o que pode gerar alterações no convívio social.

Visando promover melhor qualidade de vida e potencializar o desenvolvimento de pessoas com TEA, destaca-se a equoterapia enquanto possibilidade de intervenção.

A equoterapia utiliza o cavalo para estimular os aspectos neurosensóriomotor e psíquico. Consiste em um tipo de intervenção que exige a participação do corpo como um todo, contribuindo para o aperfeiçoamento do equilíbrio, coordenação motora, força muscular, conscientização corporal e relaxamento. Possibilita ainda, por meio da interação com o cavalo, diferentes formas de estímulo para socialização, autoestima e autoconfiança (ANDE-BRASIL, 2012).

As alterações nas habilidades motoras em crianças com TEA estão relacionadas às dificuldades de aprendizagem, as quais atingem uma população heterogênea em diferentes idades, desordens e subtipos de dificuldades.

Devido aos prejuízos no sistema de integração sensorial, crianças com TEA necessitam de estratégias e apoios específicos; as diferentes vias aferentes devem ser estimuladas de forma a favorecer o processo de aprendizagem. Neste sentido, de acordo com Klin (2006), Fittipaldi-Wert e Mowling (2009) e Schmidt (2012) crianças com TEA são favorecidas quando há estímulo visual envolvido. No entanto, Seaver e Bourret (2014) avaliaram diferentes métodos para facilitar a aprendizagem de crianças com TEA e 
http://dx.doi.org/10.5902/1984686X32575

observaram que os benefícios à população em questão podem vir de diferentes tipos de auxílio, além do visual, tais como o auxílio físico, modelo ou associação dos mesmos.

A literatura científica traz algumas pesquisas indicando a equoterapia como possibilidade de intervenção em crianças com TEA, acredita-se que a equoterapia possa promover alterações significativas em diferentes aspectos do desenvolvimento de crianças com TEA, haja vista a importância da relação entre os elementos da equoterapia na prevenção de possíveis déficits motores, visando assegurar, a longo prazo, a saúde física de crianças nessas condições (Bhat, Landa \& Galloway, 2011).

Outros estudos mencionam a melhora significativa na auto-regulação, planejamento verbal e motor, adaptação da linguagem expressiva (Gabriels et al. 2012), ganhos na coordenação corporal, força, agilidade e habilidades motoras globais (Hawkins et al. 2014), diminuição do desequilíbrio postural, melhora significativa em comportamentos adaptativos gerais, na participação com o autocuidado, nas demandas por lazer e interações (Ajzernman, Standeven, Shurtleff, 2013); diminuição dos traços apresentados pela criança com autismo (Kern et al. 2011); avanços quanto a integração sensorial, atenção direta, motivação social, sensibilidade sensorial e diminuíram a falta de atenção e a distração (Bass et al. 2009).

De forma a viabilizar uma intervenção efetiva na equoterapia, voltada à aprendizagem de posturas sobre o cavalo por crianças com TEA, faz-se necessária uma programação direcionada, visando compreender os diferentes níveis de auxílio necessários.

O objetivo do estudo foi analisar a efetividade dos níveis de auxílio visual-verbal, físico-verbal e verbal no processo de aprendizagem de posturas em equoterapia por crianças com TEA.

\section{Método}

\section{Delineamento}

O presente estudo baseou-se em delineamento de sujeito único, do tipo AB. Esse tipo de delineamento possibilita estabelecer uma relação entre a variável dependente e a variável independente em fenômenos pouco explorados (GAST; LEDFORD, 2014).

De forma a constatar se as mudanças foram decorrentes do programa experimental e não por variáveis externas à intervenção, foi realizado um estudo com réplicas em delineamentos com sujeito único (COZBY, 2011). 
http://dx.doi.org/10.5902/1984686X32575

A variável independente foi a intervenção por meio de diferentes níveis de apoio como o verbal, visual-verbal e físico-verbal; as variáveis dependentes foram a aprendizagem de posturas sobre o cavalo: montaria (M), decúbito ventral (DV), montaria lateral (ML), montaria invertida (MI), decúbito ventral invertido (DVI), em pé sobre os estribos (PE), quatro apoios (QA), quatro apoios invertido (QAI), ajoelhado (A) e ajoelhado invertido (AI).

\section{Participantes e local da pesquisa}

O estudo envolveu três participantes do gênero masculino, com faixa etária entre quatro e nove anos de idade, cuja característica comum foi o diagnóstico de Transtorno do Espectro Autista, descrição no Quadro 1.

Quadro 1- Descrição dos participantes ${ }^{1}$

\begin{tabular}{l|l|l|l|l|l|l|l|l|l}
\hline Nome & Idade & Gênero & Diagnóstico & $\begin{array}{l}\text { Idade } \\
\text { lóstico }\end{array}$ & Escola & $\begin{array}{l}\text { Outras } \\
\text { lades }\end{array}$ & Medicamentos & AE Frequência \\
\hline P1 & 9 & M & TEA & 6 & APAE & nenhuma & nenhum & 0 & $84 \%$ \\
\hline P2 & 4 & M & TEA & 3 & APAE & nenhuma & nenhum & 2 & $94 \%$ \\
\hline P3 & 5 & M & TEA & 2 & APAE & $\begin{array}{l}\text { Terapia } \\
\text { acional sem }\end{array}$ & Risperidona & 4 & $97 \%$ \\
\hline
\end{tabular}

Fonte: elaboração própria.

Os critérios de inclusão foram: apresentar diagnóstico de TEA; possuir disponibilidade para participar das sessões de equoterapia; e estar livre de contra-indicações para a equoterapia. Os critérios de exclusão foram: apresentar deficiência física ou outras doenças associadas; possuir contato prévio com as posturas sobre o cavalo; e, como critério de descontinuidade foi estabelecido número igual ou maior que três faltas consecutivas e/ou frequência inferior a $75 \%$.

A pesquisa foi realizada no Núcleo de Estudos em Atividades Físicas Adaptadas NEAFA, vinculado ao Departamento de Educação Física e Motricidade Humana - DEFMH da Universidade Federal de São Carlos - UFSCar. A intervenção em equoterapia foi desenvolvida no Centro Hípico Damha, localizado em um município de médio porte do interior paulista.

\footnotetext{
${ }^{1}$ Legenda: M= masculino, TEA (transtorno do espectro autista), APAE (Associação de Pais e Amigos dos Excepcionais), ABLA (Assessment of Basic Learning Abilities - Avaliação de Habilidades Básicas de Aprendizagem).
} 


\section{Aspectos éticos}

Atendendo a resolução 466/2012 do Conselho Nacional de Saúde, o estudo foi aprovado pelo Comitê de Ética em Pesquisas Envolvendo Seres Humanos sob o parecer n. ${ }^{\circ} 512 / 645$.

\section{Instrumentos de coleta de dados}

Anamnese: constituída por questões abertas, de modo a contemplar informações relativas à caracterização dos participantes quanto ao comportamento, linguagem falada e tipos de interesses de cada participante.

Assessment of Basic Learning Abilities (ABLA): o teste ABLA (Avaliação de Habilidades Básicas de Aprendizagem) avalia com que facilidade/dificuldade um indivíduo com desenvolvimento atípico pode aprender cinco tarefas, envolvendo diferentes tipos de controle de estímulos: Imitação Motora, Discriminação de Posição, Discriminação Visual, Escolha de Acordo com o Modelo e Discriminação Condicional Auditivo-Visual (GUILHARDI, 2003). As tarefas do ABLA são hierarquicamente organizadas de acordo com sua complexidade de controle de estímulos e têm demonstrado alta validade preditiva sobre o que o indivíduo é capaz de aprender de acordo com o desempenho no teste.

Diário de campo: esse instrumento permitiu acompanhar a evolução diária dos participantes e a descrição dos tipos de auxílio empregados durante o ensino das posturas sobre o cavalo (BARBOSA, 2013). As sessões foram registradas por meio de filmagem.

Lista de checagem: checklist para registro diário de cada um dos auxílios utilizados em cada sessão de equoterapia, ou a não realização da postura solicitada. Cada item recebeu uma pontuação para posterior análise, sendo o "auxílio verbal" $=3$, o "auxílio visual verbal"=2, o "auxílio físico"=1 e "não realiza"=0.

\section{Procedimentos de coleta de dados}

A linha de base (LB) consistiu em três sessões de equoterapia com cada uma das crianças. Durante a linha de base foram realizadas três tentativas por meio de solicitacão verbal correspondente a cada uma das posturas; uma vez que não houve resposta dos participantes aos auxílios (solicitação de execução das posturas) durante essa fase, as três sessões consecutivas de linha de base foram correspondentes a zero. 
http://dx.doi.org/10.5902/1984686X32575

Após o período de LB iniciou-se a intervenção; tanto a LB quanto a intervenção foram realizadas com o cavalo ao passo ${ }^{2}$. No momento da solicitação da postura, o cavalo permanecia parado, até que o participante assumisse a postura solicitada; a criança deveria permaner na postura por 20 segundos, após a retomada do passo pelo cavalo. $\mathrm{Na}$ sequência, foi solicitado à criança que voltasse a sentar no dorso do cavalo, retomando a postura de montaria (M) ou assumindo outra postura solicitada.

As sessões de equoterapia envolveram alternância de posturas, sendo realizadas no mínimo três e no máximo oito posturas por sessão, associadas a estratégias de reforço positivo. Após a realização da postura o participante era reforçado verbalmente (exemplo: "muito bem", "parabéns", "excelente" e "yes"), associado a um toque na mão da mediadora. Em associação ao reforço verbal, foi utilizado um outro reforçador para cada criança, de acordo com as informações fornecidas pelos pais, tais como: massinha de modelar para P1; música sertaneja para P2 e figuras dos desenhos favoritos para P3.

Cada sessão da intervenção foi constituída pelos seguintes momentos: chegada, aproximação, montaria, percurso associado às atividades específicas de trocas posturais e despedida (BARBOSA, 2013).

No decorrer da intervenção foram identificados alguns fatores limitantes para que todas as posturas fossem ensinadas a todos os participantes, devido às questões de segurança sobre o cavalo, tempo de execução e alteração do comportamento de acordo com a postura sugerida. Assim, o número de posturas apresentadas aos participantes correspondeu às possibilidades individuais $(\mathrm{P} 1=3 ; \mathrm{P} 2=6 ; \mathrm{P} 3=8)$, conforme indicado no Quadro 2.

Quadro 2- Posturas realizadas por cada um dos participantes ${ }^{3}$

\begin{tabular}{cccccccccccc}
\hline Sujeito & M & DV & ML & PE & MI & DVI & QA & QAI & A & Al & $\begin{array}{c}\text { Número de posturas } \\
\text { realizadas }\end{array}$ \\
\hline P1 & $\Omega$ & & $\Omega$ & & $\Omega$ & & & & & & 3 \\
P2 & $\Omega$ & & $\Omega$ & $\Omega$ & $\Omega$ & & & & $\Omega$ & $\Omega$ & 6 \\
P3 & $\Omega$ & $\Omega$ & $\Omega$ & $\Omega$ & $\Omega$ & $\Omega$ & $\Omega$ & $\Omega$ & & & 8 \\
\hline
\end{tabular}

Fonte: elaboração própria.

\footnotetext{
${ }^{2}$ Os cavalos executam três tipos de andaduras: passo, trote e galope. O passo caracteriza-se pelo andar natural do cavalo, sendo a passada realizada em quatro tempos.

${ }^{3}$ Legenda: $\mathrm{M}=$ montaria, $\mathrm{DV}=$ decúbito ventral, $\mathrm{ML}=$ montaria lateral, $\mathrm{MI}=$ montaria invertida, $\mathrm{DVI}=$ decúbito ventral invertido, $\mathrm{PE}=$ em pé sobre os estribos, $\mathrm{QA}=$ quatro apoios, $\mathrm{QAI}=$ quatro apoios invertido, $\mathrm{A}=$ ajoelhado e $\mathrm{AI}=$ ajoelhado invertido.
} 
http://dx.doi.org/10.5902/1984686X32575

Para o ensino das posturas foram fornecidos três tipos de auxílio aos participantes, conforme a necessidade individual, respeitando a seguinte ordem: verbal, visual-verbal e físico-verbal. No Quadro 3 é possível identificar a descrição dos possíveis auxílios fornecidos para a execução de cada uma das posturas. O número de tentativas por sessão foi regulado pelas situações naturais do ambiente.

Quadro 3- Descrição dos possíveis auxílios fornecidos em cada uma das posturas

\begin{tabular}{|c|c|c|c|}
\hline Posturas & Auxílio Verbal & $\begin{array}{c}\text { Auxílio Visual } \\
\text { associado ao Verbal }\end{array}$ & $\begin{array}{c}\text { Auxílio Físico } \\
\text { associado ao verbal }\end{array}$ \\
\hline Montaria (M) & $\begin{array}{l}\text { "passe a perna para } \\
\text { sentar de frente" }\end{array}$ & $\begin{array}{l}\text { Cartão com a postura } \\
\text { M + "passe a perna } \\
\text { para sentar de frente" }\end{array}$ & $\begin{array}{c}\text { Mão em distal de tíbia } \\
\text { ou no pé, para } \\
\text { abdução e rotação } \\
\text { externa+ "passe a } \\
\text { perna para sentar de } \\
\text { frente" }\end{array}$ \\
\hline Decúbito ventral (DV) & $\begin{array}{c}\text { "deite e abraçe o } \\
\text { cavalo" }\end{array}$ & $\begin{array}{c}\text { Cartão com a postura } \\
\text { DV + "deite e abraçe o } \\
\text { cavalo" }\end{array}$ & $\begin{array}{c}\text { Mão em região } \\
\text { torácica, inclinando o } \\
\text { corpo para frente + } \\
\text { "deite e abraçe o } \\
\text { cavalo" }\end{array}$ \\
\hline Montaria lateral (ML) & $\begin{array}{l}\text { "passe a perna para } \\
\text { sentar de lado" }\end{array}$ & $\begin{array}{l}\text { Cartão com a postura } \\
\text { ML + "passe a perna } \\
\text { para sentar de lado" }\end{array}$ & $\begin{array}{c}\text { Mão em distal de } \\
\text { fêmur, rotação externa } \\
\text { para deslocar o peso + } \\
\text { "passe a perna para } \\
\text { sentar de lado" }\end{array}$ \\
\hline Em pé no estribo (PE) & "em pé” & $\begin{array}{c}\text { Cartão com a postura } \\
+ \text { "em pé" }\end{array}$ & $\begin{array}{l}\text { Mão em proximal de } \\
\text { fêmur, realizando } \\
\text { alavanca para cima e } \\
\text { para frente + "em pé" }\end{array}$ \\
\hline Montaria invertida (MI) & $\begin{array}{l}\text { "passe a perna para } \\
\text { sentar de costas" }\end{array}$ & $\begin{array}{l}\text { Cartão com a postura } \\
\text { MI + "passe a perna } \\
\text { para sentar de costas" }\end{array}$ & $\begin{array}{c}\text { Mão em distal de tíbia } \\
\text { ou no pé, para } \\
\text { abdução e rotação } \\
\text { externa + "passe a } \\
\text { perna para sentar de } \\
\text { costas" }\end{array}$ \\
\hline $\begin{array}{l}\text { Decúbito ventral } \\
\text { invertido (DVI) }\end{array}$ & $\begin{array}{c}\text { "deite e abraçe o } \\
\text { cavalo" }\end{array}$ & $\begin{array}{c}\text { Cartão com a postura } \\
\text { DVI + "deite e abraçe o } \\
\text { cavalo" }\end{array}$ & $\begin{array}{c}\text { Mão em região } \\
\text { torácica, inclinado o } \\
\text { corpo para frente + } \\
\text { "deite e abraçe o } \\
\text { cavalo" }\end{array}$ \\
\hline Quatro apoios (QA) & $\begin{array}{l}\text { "de joelhos e mãos } \\
\text { para frente" }\end{array}$ & $\begin{array}{c}\text { Cartão com a postura } \\
\text { QA + "de joelhos e } \\
\text { mãos para frente" }\end{array}$ & $\begin{array}{c}\text { Mão na tíbia e cós da } \\
\text { calça, realizando } \\
\text { alavanca para cima + } \\
\text { "de joelhos e mãos } \\
\text { para frente" }\end{array}$ \\
\hline $\begin{array}{c}\text { Quatro apoios invertido } \\
\text { (QAI) }\end{array}$ & $\begin{array}{c}\text { "de joelhos e mãos na } \\
\text { anca do cavalo" }\end{array}$ & $\begin{array}{c}\text { Cartão com a postura } \\
\text { QAI + "De joelhos e } \\
\text { mãos na anca do } \\
\text { cavalo" }\end{array}$ & $\begin{array}{c}\text { Mão na tíbia e cós da } \\
\text { calça, realizando } \\
\text { alavanca para cima + } \\
\text { "De joelhos e mãos na } \\
\text { anca do cavalo" }\end{array}$ \\
\hline
\end{tabular}


http://dx.doi.org/10.5902/1984686X32575

\begin{tabular}{|c|c|c|c|}
\hline Ajoelhado (A) & "de joelhos" & $\begin{array}{l}\text { Cartão com a postura } \\
\text { A + "de joelhos" }\end{array}$ & $\begin{array}{l}\text { Mão no cós da calça, } \\
\text { direcionando } \\
\text { movimento para cima } \\
\text { + "de joelhos" }\end{array}$ \\
\hline $\begin{array}{l}\text { Ajoelhado invertido } \\
\text { (AI) }\end{array}$ & "de joelhos" & $\begin{array}{l}\text { Cartão com a postura } \\
\text { de Al + "de joelhos" }\end{array}$ & $\begin{array}{l}\text { Mão no cós da calça, } \\
\text { direcionando } \\
\text { movimento para cima } \\
+ \text { "de joelhos" }\end{array}$ \\
\hline
\end{tabular}

Fonte: elaboração própria.

Durante as primeiras sessões, todos os participantes receberam auxílio visual-verbal, visto que não efetuaram respostas ao estímulo verbal durante a linha de base. Durante a apresentação do auxílio visual-verbal, a mediadora observava se o participante realmente havia visualizado a imagem contida no cartão. Quando o participante não focava sua atenção na imagem, a mediadora conduzia a mão do participante até o cartão (toque assistido), de forma a direcionar o olhar da criança para a imagem. A partir do momento em que era obtida uma resposta positiva com o auxílio visual-verbal, procedia-se ao estímulo por meio de auxílio verbal.

Foram realizadas no mínimo três tentativas para cada tipo de auxílio, quando não havia resposta dos dois primeiros (visual e visual-verbal) era oferecido o auxílio físicoverbal. Após realização com sucesso da postura por meio dos auxílios visual-verbal ou físico-verbal, invertia-se a dinâmica de apresentação dos auxílios, apresentava-se primeiro o auxílio verbal, a fim de averiguar se a criança havia adquirido o repertório de modo a responder somente com o auxílio verbal.

Quando a postura solicitada era assumida pelo participante, o cavalo seguia ao passo, durante a contagem realizada pela mediadora em voz alta, até 10 (velocidade da contagem correspondente a 20 segundos), de forma a fornecer uma referência do tempo em que 0 participante deveria permanecer na postura. Ao término da contagem era solicitado que 0 participante retornasse à posição inicial, isto é, sentar sobre o dorso do cavalo quando estivesse nas posturas em DV, PE, DVI, QA, QAI, A e Al ou trocar de postura quando posicionado em M, ML e MI. As posturas foram trabalhas por 25 minutos, pois os cinco minutos antecedentes foram utilizados para estabelecer aproximação entre mediadora e participante, assim como a adaptação da criança ao cavalo e ao ambiente.

\section{Materiais}




\section{http://dx.doi.org/10.5902/1984686X32575}

Foram utilizados materiais próprios de um centro hípico e para manuseios com o cavalo, tais como rédea, estribo, cilha, manta, sela, entre outros. Também foram utilizados cartões com as imagens das posturas, cujas figuras foram compostas por fotografias da pesquisadora sobre um cavalo (Figura 1).

Figura 1- cartões com imagens das posturas (auxílio visual)

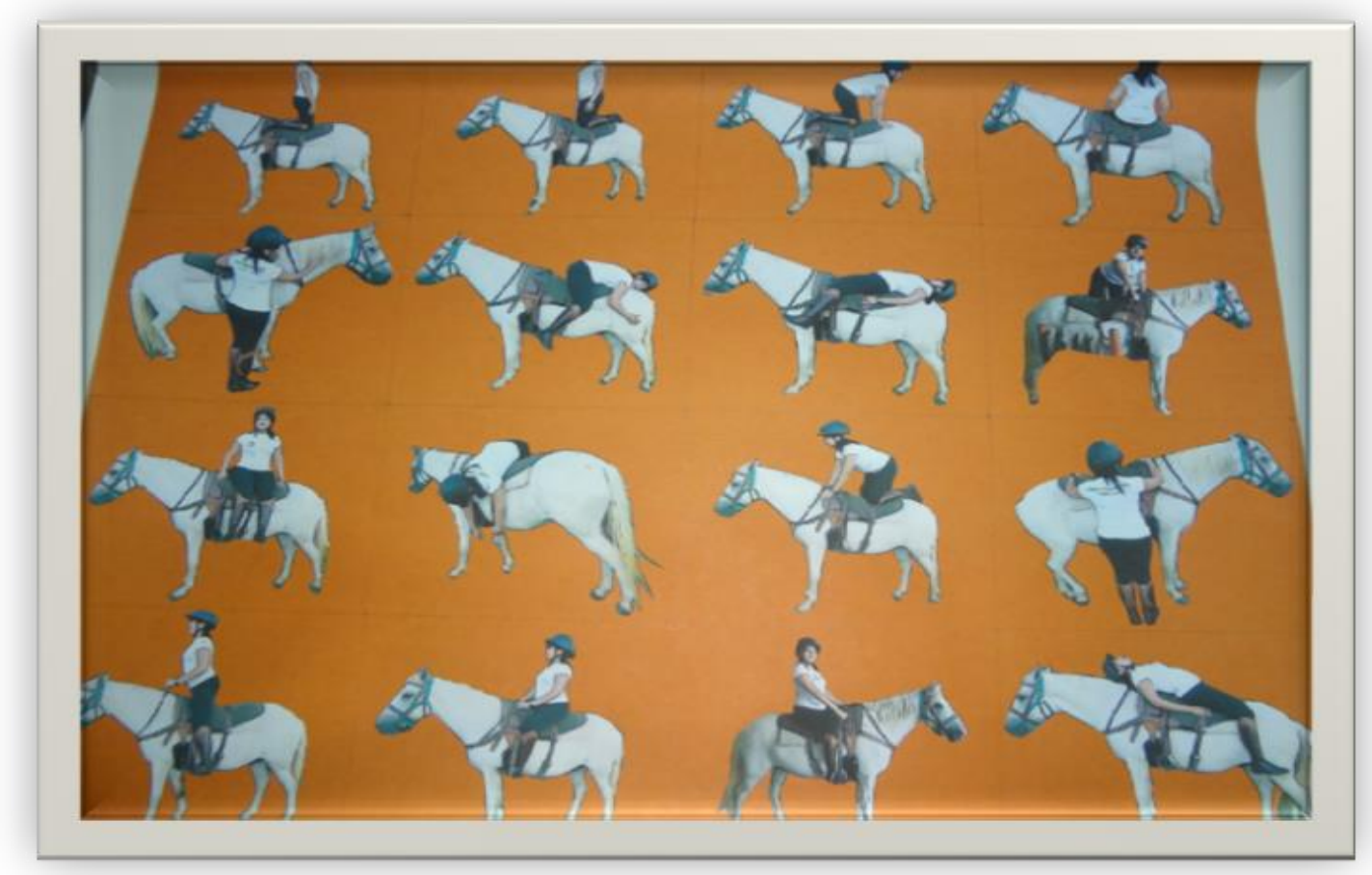

Fonte: elaboração própria.

\section{Análise dos dados}

A fim de demonstrar a evolução dos participantes em cada sessão de equoterapia, o desempenho foi calculado com base no percentual do total de pontos obtidos para a sessão em relação ao número de tentativas que surgiram no decorrer da sessão. Os dados foram representados por gráficos de barras demonstrando o desempenho do participante ao longo da intervenção.

Cada nível de auxílio recebeu uma pontuação e, quando o participante não realizava a postura solicitada, atribuía-se zero: não realiza $=0$, auxílio físico-verbal $=1$, auxílio visualverbal $=2$ e auxílio verbal $=3$.

A tabela 1 traz exemplo do cálculo da pontuação de um participante: foram oferecidas 10 tentativas para realizar a postura em montaria (M) em uma dada sessão, mas com diferentes tipos de auxílios $\left(n=n \cdot{ }^{\circ}\right.$ de tentativas $x$ pontuação correspondente ao nível de 
http://dx.doi.org/10.5902/1984686X32575

auxílio). Considerando o melhor desempenho possível (Auxílio verbal $=3$ ) em relação ao número de tentativas (10), o máximo de pontos que poderia ser atingido nessa sessão seria 30. No entanto, tendo recorrido a diferentes auxílios e considerando a possibilidade de não realização da postura pelo participante, o total de pontos obtidos foi 17, conforme indicado na Tabela 1.

Tabela 1- Exemplo de atribuição de pontuação

\begin{tabular}{|c|c|c|c|c|}
\hline $\mathrm{N}^{\circ}$ de Tentativas & & Nível de Auxílio & & Total \\
\hline 3 & $x$ & Não Realiza (0) & $=$ & 0 \\
\hline 1 & $x$ & Auxílio físico-verbal (1) & $=$ & 1 \\
\hline 2 & $x$ & Auxílio visual-verbal (2) & $=$ & 4 \\
\hline 4 & $x$ & Auxílio verbal (3) & $=$ & 12 \\
\hline Escore correspondente a & ativas & $=$ & & 17 \\
\hline
\end{tabular}

Fonte: elaboração própria.

Realizando a soma do desempenho obtido nas 10 tentativas $(0+1+4+12)$, obteve-se 17 pontos, o que corresponde a $57 \%$ do total da pontuação que poderia ser atingida. Esse cálculo foi realizado para cada uma das posturas em cada sessão. A síntese dos resultados foi obtida a partir da média de todas as sessões para cada postura, conforme o tipo de auxílio fornecido.

Para assegurar a fidedignidade dos dados, foi realizada a porcentagem de concordância interobservadores, técnica ponto a ponto, em $25 \%$ das sessões de linha de base e intervenção, selecionadas de forma aleatória para cada um dos participantes.

\section{Resultados}

Os dados referentes ao desempenho de cada um dos participantes serão apresentados a seguir.

Na Figura 2 é possível identificar o número de posturas trabalhadas com P1 (Nível ABLA 0) e visualizar o desempenho do mesmo em cada uma das três posturas conforme os tipos de auxílio ou não realização da tarefa pelo participante. da ação

Figura 2- desempenho de $\mathrm{P} 1$ em cada uma das posturas com cada tipo de auxílio ou não realização 
http://dx.doi.org/10.5902/1984686X32575

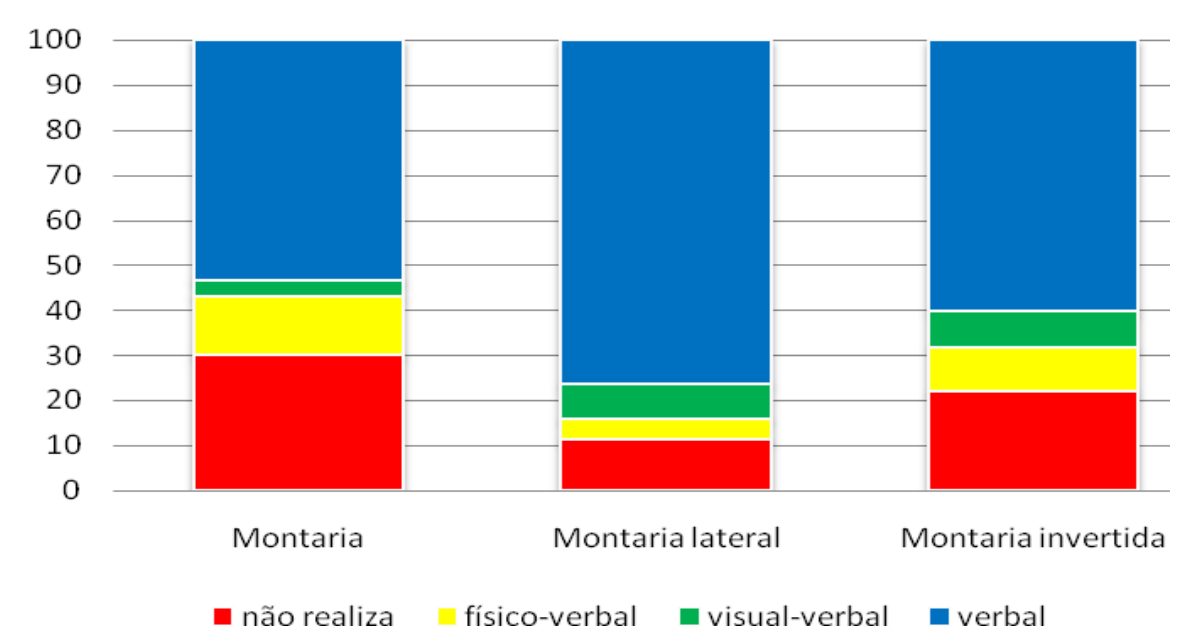

Fonte: elaboração própria.

Para P1, o melhor desempenho com auxílio exclusivamente verbal, deu-se na postura de montaria lateral (ML), seguidos de montaria invertida (MI) e montaria (M). Ainda para a postura de montaria, respondeu melhor ao auxílio físico-verbal (13\%) do que ao visualverbal (3,5\%); na montaria lateral respostas similares frente ao auxílio físico-verbal (7,64\%) e ao auxílio visual-verbal (7,84\%); na montaria invertida respondeu melhor ao auxílio físicoverbal $(9,8 \%)$ do que ao visual-verbal $(7,84 \%)$.

Na Figura 3 é possível identificar o número de posturas trabalhadas com P2 (Nível ABLA 2) e visualizar o desempenho do mesmo em cada uma das seis posturas conforme os tipos de auxílio ou não realização da tarefa pelo participante. da ação

Figura 3- desempenho de P2 em cada uma das posturas com cada tipo de auxílio ou não realização

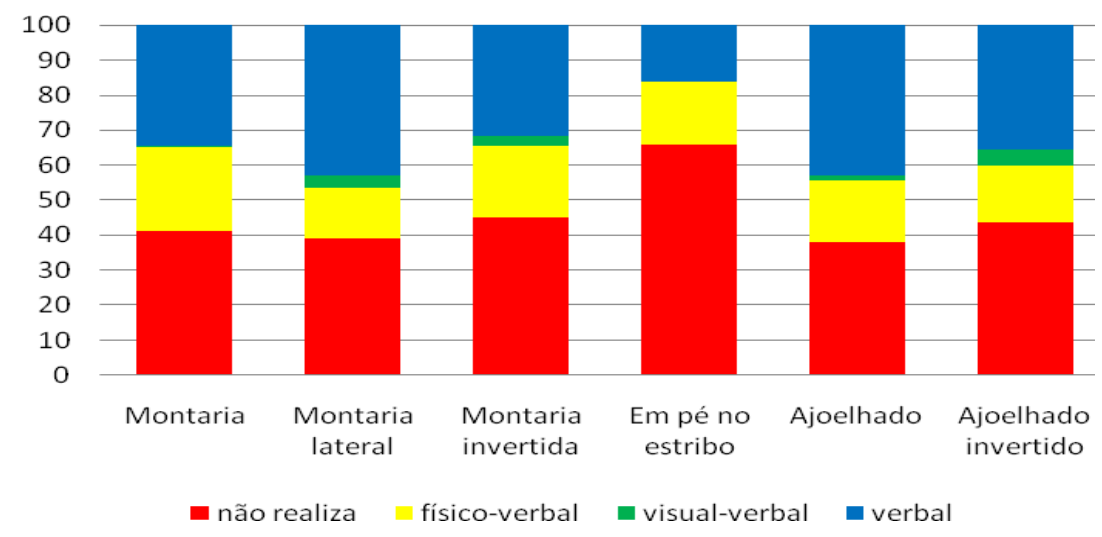

Fonte: elaboração própria.

Para P2 o melhor desempenho com auxílio exclusivamente verbal deu-se na postura ajoelhado $(A)$, seguidos de montaria $(M)$, montaria lateral $(M L)$, em pé no estribo $(P E)$, 


\section{E) REvista Educação Especial}

http://dx.doi.org/10.5902/1984686X32575

ajoelhado invertido (Al) e montaria invertida (MI). P2 respondeu melhor ao auxílio físicoverbal (média 18,37\%) do que ao visual-verbal (média 2,15\%), em todas as posturas.

$\mathrm{Na}$ Figura 4 é possível identificar o número de posturas trabalhadas com P3 (Nível ABLA 4) e visualizar o desempenho do mesmo em cada uma das oito posturas conforme os tipos de auxílio ou não realização da tarefa pelo participante

Figura 4- desempenho de P3 em cada uma das posturas com cada tipo de auxílio ou não realização da ação
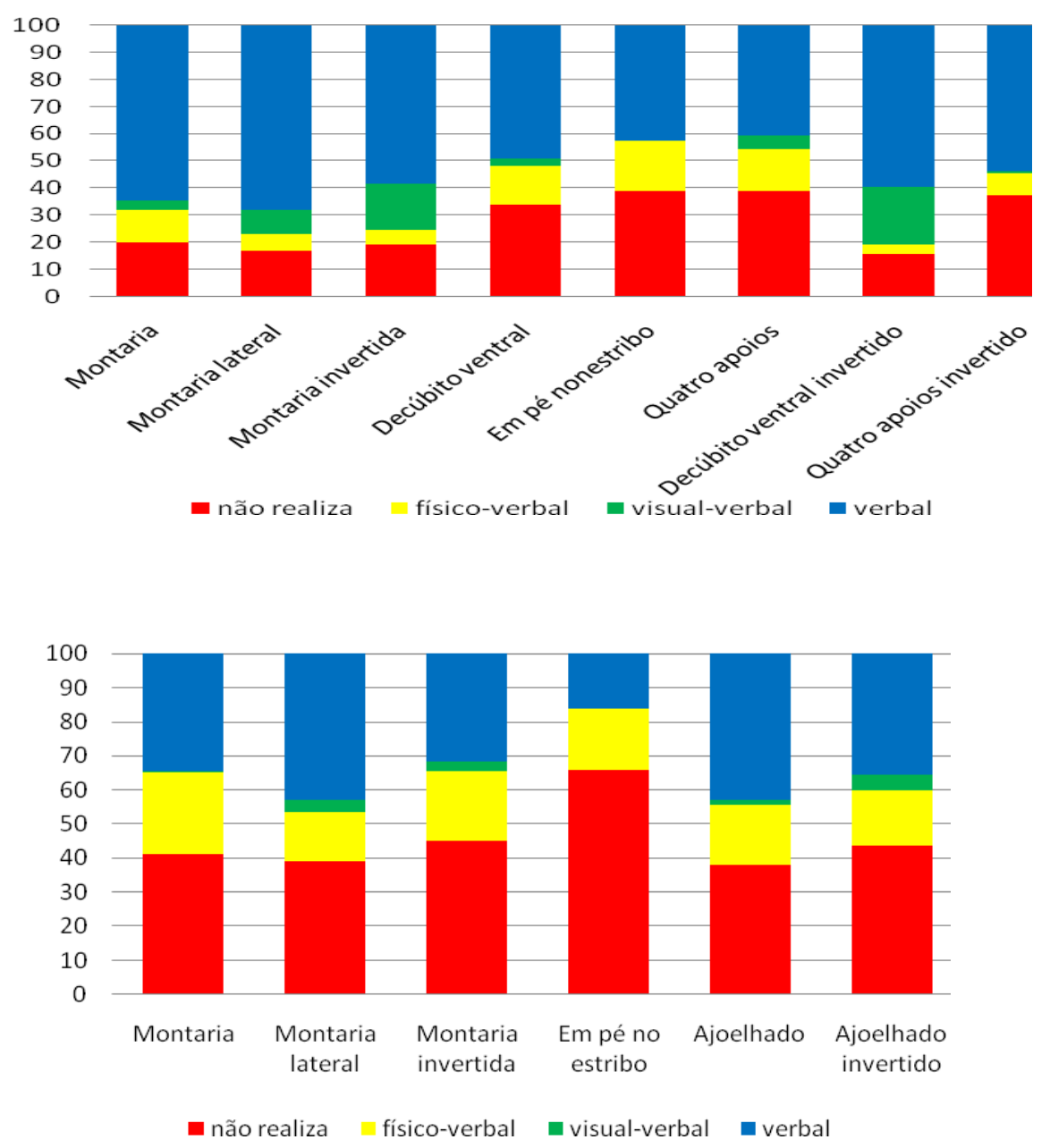

Fonte: elaboração própria.

Observou-se que, no decorrer da intervenção, também houve diferenças nas respostas frente aos diferentes tipos de auxílio em cada uma das posturas. O melhor desempenho de P3 quanto ao auxílio exclusivamente verbal deu-se na postura em montaria lateral $(M L)$, montaria $(M)$, decúbito ventral invertido (DVI), montaria invertida $(\mathrm{MI})$, quatro apoios invertido (QAI), decúbito ventral (DV), em pé no estribo (PE) e quatro apoios ( $Q A)$, respectivamente. P3 respondeu melhor ao auxílio físico-verbal, sendo este preponderante 
http://dx.doi.org/10.5902/1984686X32575

em cinco posturas (M, DV, PE, QA e QAI); em três posturas (ML, MI e DVI) houve predomínio de auxílio visual-verbal.

Em síntese, ao final da intervenção os três participantes obtiveram sucesso em executar as posturas solicitadas somente com auxílio verbal; observou-se ainda que os três participantes responderam melhor ao auxílio físico-verbal do que ao visual-verbal. Na Figura 5 observa-se a média dos auxílios para cada participante independentemente do tipo de postura e, o percentual da não realização da ação solicitada.

Figura 5- Média dos tipos de auxílios para cada participante independente do tipo de postura e a não realização da ação

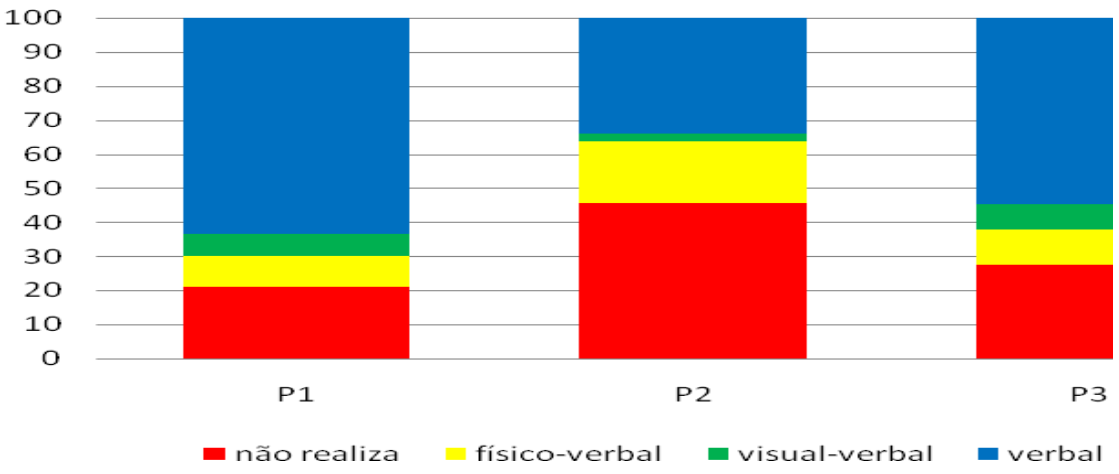

Fonte: elaboração própria.

A Figura 5 indica que o melhor desempenho frente ao auxílio exclusivamente verbal deu-se respectivamente para P1, P3 e P2, sendo P2 o participante que mais se aproveitou do auxílio físico-verbal.

\section{Discussão}

De maneira geral, P1 passou de $21 \%$ "não realiza" para "realiza com algum tipo de auxílio" em 79\% do total da intervenção; P2 evoluiu de 46\% "não realiza" para "realiza com algum tipo de auxílio" em 54\%; e P3 avançou de 28\% "não realiza" para "realiza com algum tipo de auxílio" em 72\% da intervenção (Figura 5). Além disso, observou-se correlação entre o nível $A B L A$ dos participantes e o aproveitamento na aprendizagem das posturas: 0 participante com maior nível ABLA (P3) foi capaz de realizar maior número de posturas (8), enquanto o participante com menor nível $A B L A(P 1)$ obteve sucesso em um menor número de posturas (3). No estudo de Martin et al. (2008) os autores apontaram que o desempenho no ABLA tem elevada validade preditiva para os tipos de tarefas que um indivíduo será capaz de aprender. Além disso, também observaram que, quanto melhor o nível ABLA, 
http://dx.doi.org/10.5902/1984686X32575

menos comportamentos aberrantes são manifestados no decorrer das tarefas; verificaram ainda que o desempenho no ABLA é um indicador da capacidade de aprendizado, como também pode prever a eficácia na resposta a diferentes estímulos.

Quanto ao processo de aprendizagem referente à efetividade dos níveis de auxílio, notou-se que não houve linearidade para nenhum dos participantes quanto ao aprendizado das posturas por meio dos auxílios visual-verbal e físico-verbal ou vice e versa. Almeida (2012) aponta que há basicamente três estilos de aprendizagem: visual, auditiva e cinestésica; na aprendizagem visual o processo dá-se por meio da leitura ou visualização de uma imagem, na auditiva se refere a instruções por meio da verbalização e a cinestésica é quando a aprendizagem ocorre por meio do movimento, assim observa-se que, de maneira geral, os participantes do estudo se beneficiaram dos três estilos indistintamente.

No entanto, quanto à aquisição do aprendizado somente com o auxílio verbal foi conquistado por P1, P2 e P3 de maneira crescente no decorrer das sessões; A Tabela 2 ilustra a evolução dos participantes quanto ao nível de auxílios ao longo da intervenção.

Tabela 2- Evolução dos participantes quanto ao nível de auxílio

\begin{tabular}{cccccccc}
\hline & Não realiza & & Físico verbal & & Visual verbal & & Verbal \\
\hline P1 & $21 \%$ & $\rightarrow$ & $9 \%$ & $\rightarrow$ & $6 \%$ & $\rightarrow$ & $64 \%$ \\
P2 & $46 \%$ & $\rightarrow$ & $18 \%$ & $\rightarrow$ & $2 \%$ & $\rightarrow$ & $34 \%$ \\
P3 & $28 \%$ & $\rightarrow$ & $10 \%$ & $\rightarrow$ & $7 \%$ & $\rightarrow$ & $55 \%$ \\
\hline
\end{tabular}

Fonte: Elaboração própria.

Os resultados indicam que, para os três participantes, houve diferenças idiossincráticas quanto à sensibilidade frente a cada tipo de auxílio. Estes resultados culminam com os apontamentos de Seaver e Bourret (2014), que observaram que procedimentos distintos podem viabilizar a aprendizagem e ser diferencialmente eficiente entre indivíduos.

Deste modo, constatou-se que foi necessária a combinação de diferentes tipos de auxílio para ensinar posturas, ou seja, a resposta ao estímulo exclusivamente verbal contou com a associação prévia dos auxílios visual-verbal e físico-verbal, em diferentes proporções, durante o processo de aprendizagem das várias posturas.

Destaca-se ainda que nem todos os participantes responderam da mesma forma frente aos mesmos tipos de auxílio, necessitando de diferentes tipos de estímulos em cada postura. Sendo assim, para P1 a associação dos auxílios físico-verbal e visual-verbal ocorreu nas três posturas, porém foi mais evidente na postura em montaria lateral (7,64\%) e montaria invertida $(7,84 \%)$ do que na postura em montaria (3,5\%) (Figura 2); para P2 a 
associação desses mesmos auxílios foi pouco perceptível, o auxílio visual-verbal apresentou-se discretamente nas posturas em montaria lateral $(3,8 \%)$, montaria invertida $(2,57 \%)$, ajoelhado (1,4\%) e ajoelhado invertido (4,85\%) (Figura 3); para P3 o responder com auxílio visual-verbal foi mais evidente nas posturas em e decúbito ventral invertido $(21,1 \%)$, montaria invertida (16,95\%), montaria lateral $(9 \%)$ e houve uma discreta associação do auxílio visual-verbal em decúbito ventral (5\%) (Figura 4).

Desta forma, o estudo demonstra que a associação dos auxílios visual-verbal e físicoverbal não foram tão evidentes no sentido de afirmar que foi a associação dos estímulos que favoreceu a aprendizagem das posturas evoluindo para o auxílio exclusivamente verbal. Os dados encontrados nesse estudo convergem aos apresentados por Walls et al. (1979), que compararam dicas físicas, modelo, verbal e combinação das três dicas no ensino de tarefas de montagem de um motor. Os autores verificaram que o estímulo verbal foi associado com o maior número de erros, seguidos pelas dicas de modelo, auxílio físico e a combinação das mesmas, ou seja, quando as dicas foram combinadas a aprendizagem foi favorecida.

Em nossa pesquisa observou-se que, apesar da oportunidade de associação de auxílios, P1, P2 e P3 responderam melhor ao físico-verbal para alcançar somente o verbal, nos resultados Walls e colaboradores (1979), os achados indicaram que a efetividade de uma intervenção para um indivíduo pode não ser tão adequada para outro. Assim, observase que fatores individuais como cognição e atenção mediam a compreensão da criança e consequentemente a efetividade da estratégia proposta.

Apesar da literatura científica enfatizar que os indivíduos com TEA são, em sua maioria, pensadores visuais (KLIN, 2006; FITTIPALDI-WERT; MOWLING, 2009; SCHMIDT, 2012), verificou-se que não houve predomínio do auxílio visual-verbal sobre os demais, conforme a hipótese inicial. Os dados encontrados no presente estudo reforçam a necessidade do respeito às diferenças individuais apresentadas pelas crianças com TEA, levando à necessidade de individualização do processo de aprendizagem. Silva e Balbino (2015) abordam que crianças com TEA possuem peculiaridades e respostas diferenciadas frente às atividades, apontando ainda que a escolha correta de estratégias adaptadas é de suma importância para o sucesso na aprendizagem. Na mesma direção, Almeida (2012) aponta que o estilo de aprendizagem deve ser ajustado a cada criança com TEA pois, se a criança for mais visual, pode-se mostrar a imagem ou o objeto pretendido à aprendizagem, para que possa ajudá-la a compreender o que está sendo solicitado; no entanto, se a 
http://dx.doi.org/10.5902/1984686X32575

criança for predominantemente cinestésica deve-se oportunizar a percepção do movimento ou direcionar o mesmo para favorecer a assimilação.

O emprego do auxílio visual-verbal demonstrou maior eficiência no participante com melhor habilidade no ABLA (P3). No estudo de Fetsch, DeAngelis e Angelaki (2010) os autores esclarecem a relação entre a percepção visual e o sistema vestibular, onde a percepção do movimento é induzida pelo movimento visual. Assim, a auto-percepção do movimento é um desafio para o sistema nervoso central, em grande parte porque envolve a integração de um conjunto complexo de sinais sensoriais visuais e não visuais.

Dos três participantes do estudo, P3 foi o que apresentou maior facilidade para focar nas dicas visuo-verbais e demonstrou maior agilidade para realizar as posturas solicitadas, permitindo que um número maior de posturas pudessem ser alcançadas com sucesso na aprendizagem. De acordo com os dados apresentados por P3, pode-se inferir que há uma melhor integridade do sistema sensorial desse participante em relação aos demais, o que poderia explicar o melhor aproveitamento do estímulo visual-verbal e, consequentemente, maior desenvoltura na aprendizagem das posturas.

\section{Conclusão}

De maneira geral, os auxílios foram efetivos para ensinar diferentes posturas sobre o cavalo, visto que os três participantes apresentaram evolução na aprendizagem, em relação à linha de base.

Quanto às estratégias de auxílios verbal, visual-verbal e físico-verbal, buscou-se associar os estímulos visual e físico ao verbal, de modo a proporcionar uma relação entre as diferentes entradas sensoriais às crianças com TEA, a fim de aprimorar o aprendizado.

No tocante à aprendizagem das posturas e a evolução das mesmas quanto ao tipo de auxílio oferecido, observou-se que todos os participantes passaram a responder somente com o auxílio verbal ao final da intervenção, sendo que responderam com maior efetividade ao auxílio físico-verbal em comparação ao visual-verbal, para posteriormente responderem somente ao auxílio verbal. Os resultados indicam que os níveis de auxílio necessários à aprendizagem de posturas em equoterapia devem variar conforme as necessidades individuais, não havendo um único método eficiente; todavia observa-se que a associação de estímulos de maneira direcionada pode levar ao sucesso na aprendizagem. Cada criança apresenta uma maneira peculiar de se relacionar com 0 mundo $e$, consequentemente com os processos de aprendizagem; de acordo com o contexto e a tarefada solicitada. 
http://dx.doi.org/10.5902/1984686X32575

Em estudos futuros, sugere-se que as pesquisas contemplem maior número de sujeitos e maior tempo de intervenção.

\section{Referências}

AJZENMAN, H. F., STANDEVEN, J. W., SHURTLEFF, T. L. Effect of hippotherapy on motor control, adaptive behaviors, and participation in children with autism spectrum disorder: a pilot study. American Journal of Occupational Therapy, v.67, 653-663, 2013.

ANDE-BRASIL, 2. Equoterapia: considerações complementares. Disponível em: <http: //www.equoterapia.org.br/site/equoterapia.php>, 2012. Acesso em: 08 novembro 2012.

ALMEIDA, A. Como ensinar crianças autistas, de 21 de abril de 2012. Disponível em: < http://pedagogiaaopedaletra.com/como-ensinar-criancas-autistas/. Acesso em 26 agosto 2016.

BARBOSA, G. O. Efeito de um programa de equoterapia nos aspectos psicomotores de crianças com indicativos do TDAH. 2005. 193 f. Dissertação (Mestrado em Educação Especial) - Centro de Educação e Ciências Humanas, Universidade Federal de São Carlos. São Carlos, 2013.

BASS, M. B. DUCHOWNY, C. A., \& LLABRE, M. M. The effect of therapeutic horseback riding on social functioning of children with autism. Journal of Autism and Developmental Disorders, v. 39, 1261-1267, 2009.

BHAT, A.; LANDA, R.; GALLOWAY, J. Current perspectives on motor functioning in infants children, and adults with Autism Spectrum Disorders. Physical Therapy, v. 91, pp. 1116$1129,2011$.

COZBY, P. C. Métodos de Pesquisa em Ciências do Comportamento. São Paulo: Atlas, 2011. 238-249 p.

DSM 5. Diagnostic and Statistical Manual of Mental Disorders. 5 edição. [S.I.]: [s.n.], 2013.

FETSCH, C. R.; DEANGELIS, G. C.; ANGELAKI, D. E. REVIEW ARTICLE: Visualvestibular cue integration for heading perception: applications of optimal cue integration theory. European Journal of Neuroscience, v. 31, pp. 1721-1729, 2010.

FITTIPALDI-WERT, J., MOWLING, C. Using visual supports for students with autism in physical education. JOPERD, v. 80, n. 2, p. 39-48, 2009.

GABRIELS, R. L., AGNEW, J. A.,HOLT, K. D., SHOFFNER, A. ZHAOZING, P.,RUZZANO, $S$., et al. Pilot study measuring the effects of therapeutic horseback riding on school-age 
http://dx.doi.org/10.5902/1984686X32575

children and adolescents with autism spectrum disorders. Research in Autism Spectrum Disorders, v.6, 578-588, 2012.

GAST, D. L.; LEDFORD, J. R. Single case research methodology: applications in special education and behavioral sciences. 2 ed. New York: Routledge, 2014, 462 p.

GUILHARDI, C. Potencial preditivo do teste ABLA na aquisição de treinos de discriminações condicionais auditivo-visuais e teste de outras discriminações condicionais. 2003. 245 f. Dissertação (Mestrado em Psicologia) - Pontifícia Universidade Católica de São Paulo, São Paulo, 2003.

HAWKINS, B. L., RYAN, J. B., DONALDSON, M. C. Effects of equine-assisted therapy on Gross Motor Skills of Two Children with Autism Spectrum Disorder. Therapeutic Recreation Journal, v.48, 135-149, 2014.

KERN, J., FletCheR, C., GARVER, C., MEHTA, J., GRANNEMANN, B., KNOX, K., RICHARDSON, T. Y TRIVEDI, M. Prospective Trial of Equine-assisted Activities in Autism Spectrum Disorder. Alernative Therapies in Health y Medicine, v.17, 14-20, 2011.

KLIN, A.; CHAWARSKA, K.; RUBIN, E.; VOLKMAR, F. Avaliação clínica de crianças com risco de autismo. Educação, Porto Alegre, v.58, n.1, p.255-297, 2006.

MARTIN, G. L.; THORSTEINSSON, J. R.; YU, C. T.; MARTIN, T. L.; VAUSE, T. The assessment of basic learning abilities test for predicting learning of persons with intellectual disabilities. Behavior Modification, v. 32, n. 2, p.228-247, 2008.

SCHMIDT, C. Temple Grandin e o autismo: uma análise do filme. Revista Brasileira de Educação Especial, v.18, n. 2, p. 179-194, 2012.

SEAVER, J. L.; BOURRET, J. C. An evaluation of response prompts for teaching behavior chains. Journal of applied behavior analysis, v. 47, n. 4, p. 777-792, 2014.

SILVA, M.K.; BALBINO, E. S. A importância da formação do professor frente ao transtorno do espectro autista - tea: estratégias educativas adaptadas. In: Encontro ALAGOANO DE EDUCAÇÃO INCLUSIVA, 6. Universidade Federal de Alagoas, dezembro 2015.

WALLS, R. T.; ELLIS, W. D.; ZANE, T.; VANDERPOEL, S. J. Tactile, auditory, and visual prompting in teaching complex assembly tasks. Education and Training of the Mentally Retarded, v.14, n.2, 120-130, 1979.

WALTER, C. C. F. Avaliação de um programa de comunicação alternativa e ampliada para mães de adolescentes com autismo. 2006. 137 f. Tese (Doutorado em Educação Especial) - Centro de Educação e Ciências Humanas, Universidade Federal de São Carlos. São Carlos, 2006. 


\section{Correspondência}

Gardenia de Oliveira Barbosa - Universidade Federal de São Carlos, Centro de Educação e Ciências Humanas, Departamento de Psicologia. Rodovia Washington Luís, Km 235. Monjolinho. CEP: 13565-905. São Carlos, São Paulo, Brasil. http://orcid.org/0000-0002-1825-0097

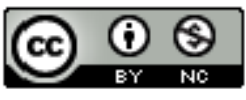

This work is licensed under a Creative Commons Attribution-NonCommercial 4.0 International (CC BY-NC 4.0) 